\title{
Magnetization Phenomenon for Variable Flux Memory Motor using Finite Element Method and Experimental Verification
}

\author{
Young Hyun Kim and Jung Ho Lee* \\ Department of Electrical Engineering, Hanbat National University, Daejeon, Korea
}

(Received 5 September 2016, Received in final form 26 October 2016, Accepted 28 October 2016)

\begin{abstract}
In this study, we evaluate the performance of permanent magnets (PMs). The efficiency of attraction in the high speed region was studied using the Variable Flux Memory Motor (VFMM). It is presented in order to analyze the magnetic characteristics of PMs, using the second quadrant plan data with re- and demagnetization. In addition, this study focuses on the evaluation of operational characteristics relative to the magnetizing directions according to the d-axis currents, by using one of the finite element solutions. The feasibility of application for the VFMM has been experimentally demonstrated.
\end{abstract}

Keywords : FEM, Re- and Demagnetization, VFMM

\section{Introduction}

In order to cope with the challenges of environmental protection and energy shortage, researchers have been making technological progress and developing new concepts of high efficiency and energy saving. In particular, in an effort to reduce the power consumption of electrical machines, many engineers have been conducting research on the development of high efficiency machines. There has been a particular interest in permanent magnet synchronous motors (PMSMs), which rely on field-weakening control, as they operate with high torque characteristics, high efficiency, and a wide range of operating regions [1-3].

However, if a PMSM operates at high speed and high power, the back electromotive force (back-EMF) will exceed the DC link voltage of the inverter [4]. Moreover, a field-weakening control is required to increase the negative d-axis current, $i_{d}$, in order to use the high speed regions where efficiency is decreased. In order to solve this issue, studying memory motors is necessary in order to increase efficiency through a wide region of operation.

Memory motors can be built as either variable-flux or pole-changing machines [5]. They utilize a flux-concentration principle that allows the generation of air-gap flux

(C)The Korean Magnetics Society. All rights reserved.

*Corresponding author: Tel: +82-42-821-1098

Fax: +82-42-821-1088, e-mail: limotor@hanbat.ac.kr densities typical for high-efficiency machines. In both types of machines, the magnetization of permanent magnets (PMs) can be simply varied by a short current pulse, without the need of a permanent de-magnetizing current as in conventional internal PM machines with a fieldweakening control. The unique features of the variableflux memory motor (VFMM) include the air-gap flux created by rotor magnets, which can be continuously varied within a fraction of the period of the stator current, and the re-magnetization current, which becomes possible without additional loss because the period of time through the stator windings is very short, which dissipates negligible losses. Previous studies showed that the load current, $i_{q}$, would not de-magnetize the magnets [6]. The effects of magnetization are important in the performance of a VFMM. In this study, we proposed a method for checking the operational principle and machine control under reand de-magnetization conditions. The effect of the applied $\mathrm{d}$-axis currents, $i_{d}$, was studied using the finite element method (FEM). Additionally, this analysis demonstrated the various aspects of change of back-EMF, which was also confirmed by experiments.

\section{Principle and Analytical Modeling of a VFMM}

\subsection{Operational Principles of a VFMM}

The VFMM is used in many home appliances including washing machines and cooling machines. Table 1 shows 
Table 1. Specifications for the design model.

\begin{tabular}{lcc}
\hline \hline \multicolumn{1}{c}{ Parameters } & Value & Unit \\
\hline Phase & 3 & - \\
Poles / Slots & $4 / 24$ & - \\
Rated Power & 340 & {$[\mathrm{~W}]$} \\
Rated Torque & 0.9 & {$[\mathrm{Nm}]$} \\
Rated Speed & 3600 & {$[\mathrm{rpm}]$} \\
DC link Voltage & 310 & {$\left[\mathrm{~V}_{\mathrm{dc}}\right]$} \\
Turns per Phase & 44 & - \\
Out Diameter & 122 & {$[\mathrm{~mm}]$} \\
Airgap Size & 0.4 & {$[\mathrm{~mm}]$} \\
\hline
\end{tabular}

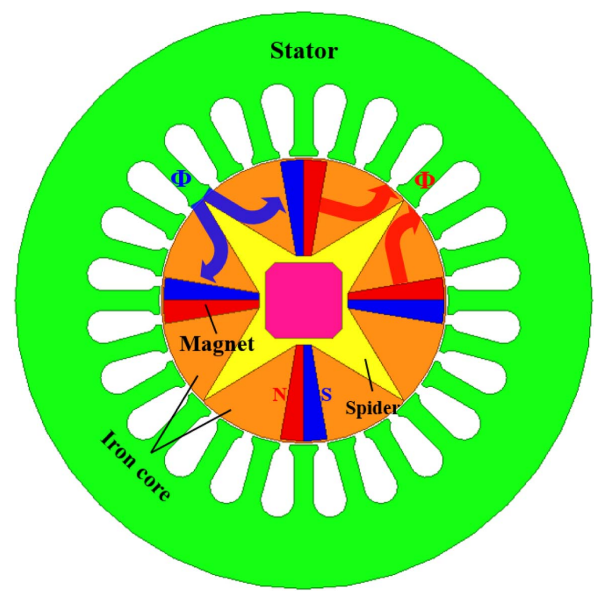

Fig. 1. (Color online) 3-D structure view of four-pole VFMM.

the design specification for a varying-speed cooling system. Figure 1 shows the detailed structure of a four-pole VFMM model. To obtain a guarantee of the effects of the re- and de-magnetization current, we have adopted trapezoidal rotor magnets, which can be spatially controlled. Tangentially magnetized rotor magnets with N-poles and S-poles generate flux, $\phi_{m}$, through the air gap into the stator. After applying a short pulse of the stator current, $i_{d}$, in a counter direction, the rotor magnets are partially demagnetized, as shown in Fig. 2(a) and (b). This demagnetized point in the magnets is called the zero flux radius, $r_{0}[6]$.

\subsection{Analytical Modeling of a VFMM}

Figure 3 shows the half-pole model of a VFMM rotor. In the figure, the following notations were used:

$d_{1}, d_{2}$ : PM width on the shaft and air-gap side, respectively.

$b_{m}$ : magnet radial length.

$x$ : circumferential coordinate.

In addition, we denote the remnant flux density of the magnet as $B_{r}$, its coercive force as $H_{c}$, the air-gap flux

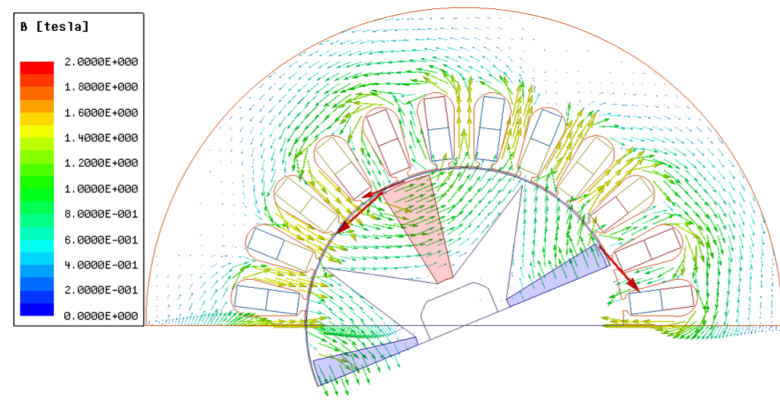

(a)

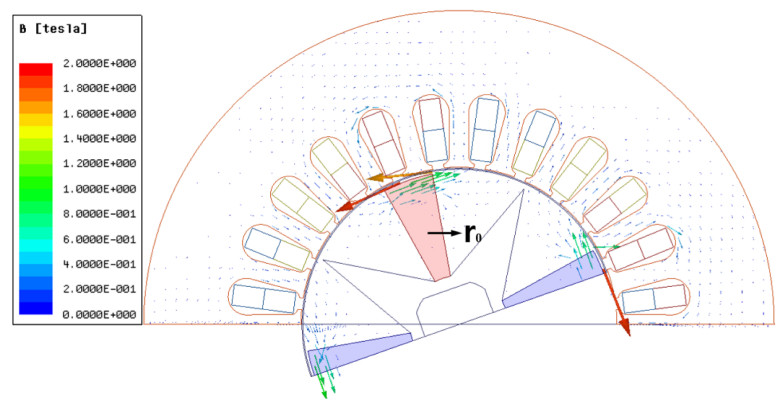

(b)

Fig. 2. (Color online) Magnetic phenomenon [(a) fully magnetized VFMM (b) partially].

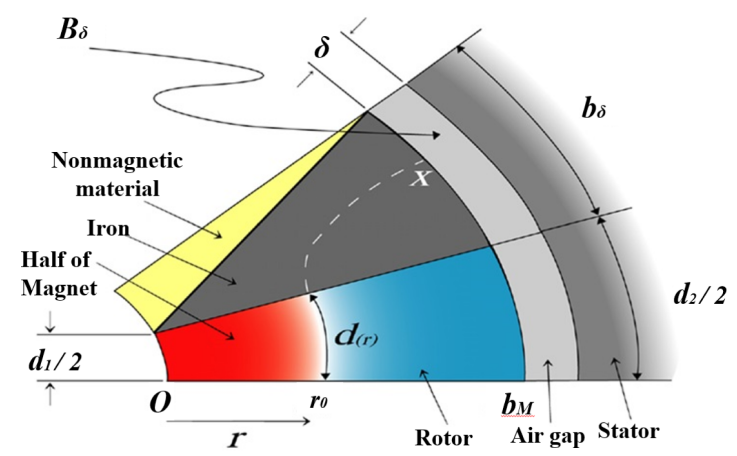

Fig. 3. (Color online) Cross-sectional view of the half pole of VFMM rotor.

density as $B_{\delta}$, and the machine length as $l$. As stated above, the equations of $B_{\delta}, d_{2}$ and $x$ are well known from previously published papers [5].

2.2.1. Condition of the maximum de-magnetization effect

If an intense short-pulse current is applied to the PMs reversely, the magnetization direction of the PMs will remain changed. When the air-gap flux density, $B_{\delta}$, is maximized, the condition $x=B_{\delta}$ would be substituted for $r=b_{m}$ in the flux contour equation. In this case, the coordinates of $x$ and $b_{\delta}$ are described as follows: 


$$
\begin{aligned}
& b_{\delta}=\frac{1}{2} \tau_{\rho}-d_{2}=\tau_{\rho}\left(\frac{1}{2}-d_{2}^{\prime}\right) \\
& x=b_{\delta}=\tau_{\rho}\left(\frac{1}{2}-d_{2}^{\prime}\right)
\end{aligned}
$$

which leads to the expression of the air-gap flux density, $B_{\delta \max }$, as below:

$$
B_{\delta \max }=\frac{B_{r}}{\frac{B_{r}}{\mu_{0} H_{c}} \times \frac{\delta / \tau_{\rho}}{d_{2}{ }^{\prime}-d_{1} / \tau_{\rho}} \ln \left(\frac{d_{2}{ }^{\prime}-d_{1} / \tau_{\rho}}{d_{1} / \tau_{\rho}} \frac{\tau}{b_{M}}+1\right)+\frac{2 \pi}{\rho c}\left(\frac{1}{2}-d_{2}{ }^{\prime}\right)}
$$

The condition of parameter $r_{0}^{\prime}$, which is the maximum air-gap flux density for the occurrence of flow, is related to the coordinates of $x$ in the following way:

$$
\begin{aligned}
& x=\frac{2 \pi}{\rho}\left(\frac{1}{2}-d_{2}{ }^{\prime}\right) \\
& r_{0}{ }^{\prime}=\frac{r_{0}}{\tau_{\rho}}=\frac{b_{M}}{\tau_{\rho}}=\frac{r}{l}
\end{aligned}
$$

2.2.2. Condition of half of the de-magnetization effect If half of the de-magnetization effect occurs, the routes $x$ and $b_{\delta}$ of the flux are changed as follows:

$$
\begin{aligned}
& b_{\delta}=\frac{1}{2} \tau_{\rho}-d_{2}=\tau_{\rho}\left(\frac{1}{2}-d_{2}{ }^{\prime}\right) \\
& x=\frac{1}{2} b_{\delta}=\frac{\tau_{\rho}}{2}\left(\frac{1}{2}-d_{2}{ }^{\prime}\right)
\end{aligned}
$$
which leads to the expression of flux density $B_{\frac{1}{2} \delta}$ as
given below:

$$
B_{\frac{1}{2} \delta}=\frac{B_{r}}{2 \frac{B_{r}}{\mu_{0} H_{c}} \times \frac{\delta}{d_{2}-d_{1}} \ln \left(\frac{d_{2}-d_{1}}{d_{1}} \frac{r}{b_{M}}+1\right)+\frac{\pi}{\rho c}\left(\frac{1}{2}-d_{2}{ }^{\prime}\right)}
$$

One can further state that:

$$
x=\frac{2 \pi}{4 \rho}\left(\frac{1}{2}-d_{2}{ }^{\prime}\right)
$$

The condition of the parameter $r_{0}^{\prime}$, which is the maximum air-gap flux density for the occurrence of flow, is related to the coordinates of $x$ in the following way:

$$
r_{0}{ }^{\prime}=\frac{r_{0}}{\tau_{\rho}}=\frac{b_{M}}{2 \tau_{\rho}}=\frac{r}{2 l}
$$

\section{FEM Analysis Descriptions}

\subsection{Analysis of Re- and De-magnetization Character-} istics

The PM machines are designed to avoid the irreversible de-magnetization phenomenon caused by an external magnetic field.

However, the VFMM utilizes this simple re- and demagnetization phenomenon, and therefore, it should be used with PMs of a small coercivity. Figure 4 shows the de-magnetization curve (B-H) of an Alnico9 on the second quadrant plane. The characteristics of the $\mathrm{PM}$ are represented by the second quadrant of the $\mathrm{B}-\mathrm{H}$ curve. The conventional analysis of FEMs is applied as a straight line in the second quadrant of any two of the parameters: $B_{r}$, $H_{c}$, or $\mu_{s}$. However, with characteristics such as dependent temperature or external magnetic fields, B-H data is required for a non-linear magnet. When the negative $\mathrm{d}$ axis current is applied as an opposite magnetic field, the operating point "P1" moves to "P2".

If the operating point does not reach a value below the knee point, it returns to the "P1" point. If, however, an intensity-negative d-axis current is applied, the $B_{r}$ will be reduced to a value lower than that of the knee point [7]. Figure 5 shows the flux-flow patterns with respect to the d-axis current. Figure 5(a) shows the magnetic flux patterns for fully magnetized rotors, and Fig. 5(b) shows the patterns for the partially magnetized rotors. The magnetization direction of the magnetic flux pattern in the initial magnetization state is formed from left to right. However, the magnetization direction is changed from the surface close to the shaft by the application of a d-axis pulse current. As a result, a zero flux radius, $r_{0}$, is gene-

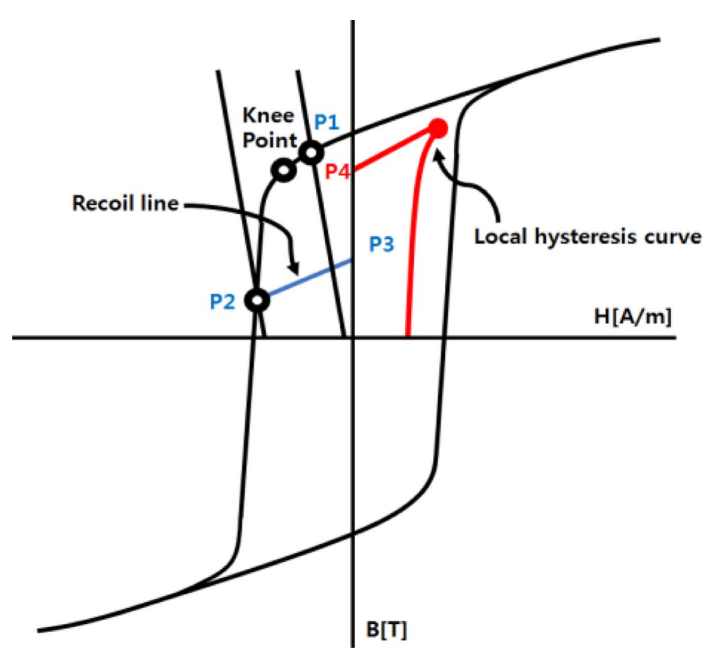

Fig. 4. (Color online) Demagnetization curve of an alnico9 on second quadrant plane. 


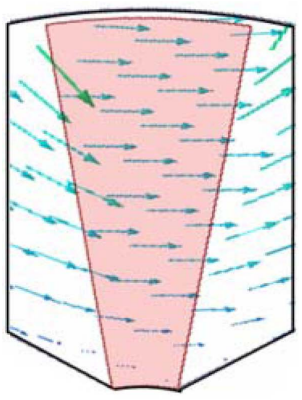

(a)

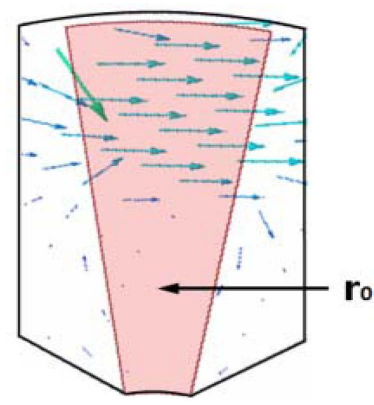

(b)
Fig. 5. (Color online) Magnetization directions and flux flow pattern of [(a) fully magnetized magnet (b) demagnetized magnet].

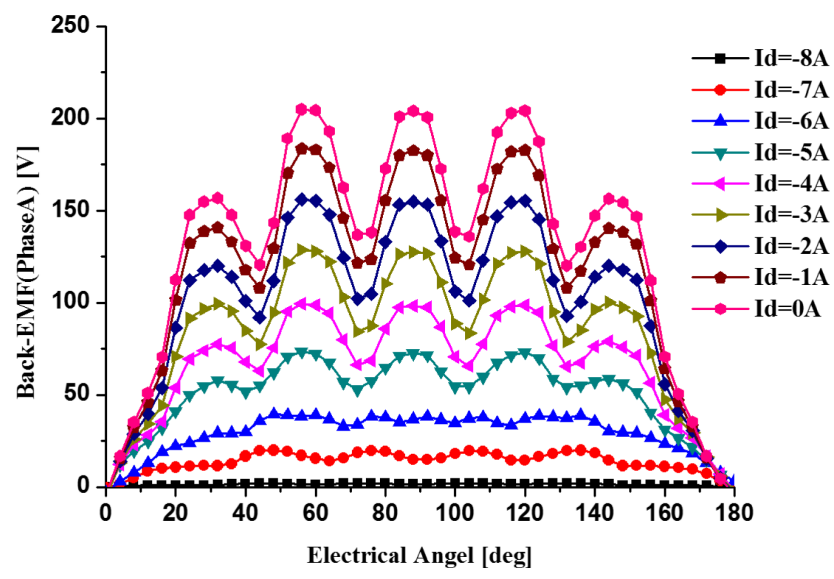

Fig. 6. (Color online) Comparison of back-EMF waves according to the d-axis currents.

rated. Further, rather than having to use a complex equation to determine the magnitude of the magneto-motive force
(MMF) in order to determine the position of $r_{0}$, the position variation can be accurately and conveniently calculated based on the increasing de-magnetization of the MMF. A reverse magnetic force is also expected to be generated, which by increasing the d-axis current, achieves a value higher than the amount of magnetization of the magnet.

Figure 6 shows the back-EMF waves induced at a consistently decreasing ratio. If the de-magnetizing condition is set at $-7 \mathrm{~A}$ for the d-axis current, the back-EMF is reduced by approximately $91.3 \%$ from the value of 147.3 $\mathrm{V}$ to $12.8 \mathrm{~V}$.

Figure 7 shows the back-EMF wave and de-magnetization current. This is intended to represent the generated back-EMF before and after the application of the d-axis current.

\section{Control Principle of VFMM}

If PM machines operate at high speed and high power, the back-EMF cannot exceed the DC link voltage of an inverter. However, a field-weakening control is required to increase the d-axis current and reduce the back-EMF, so the high speed regions can be utilized, where the efficiency of the machine would increase.

On the other hand, through the VFMM, air-gap flux is created by rotor magnets, which can be continuously varied within a fraction of the period of the stator current, as well as the re-magnetization current which is possible to supply without additional loss.

A VFMM has a back-EMF control capability similar to a wound rotor machine, but with an important difference: a wound rotor machine demands the losses of a per-

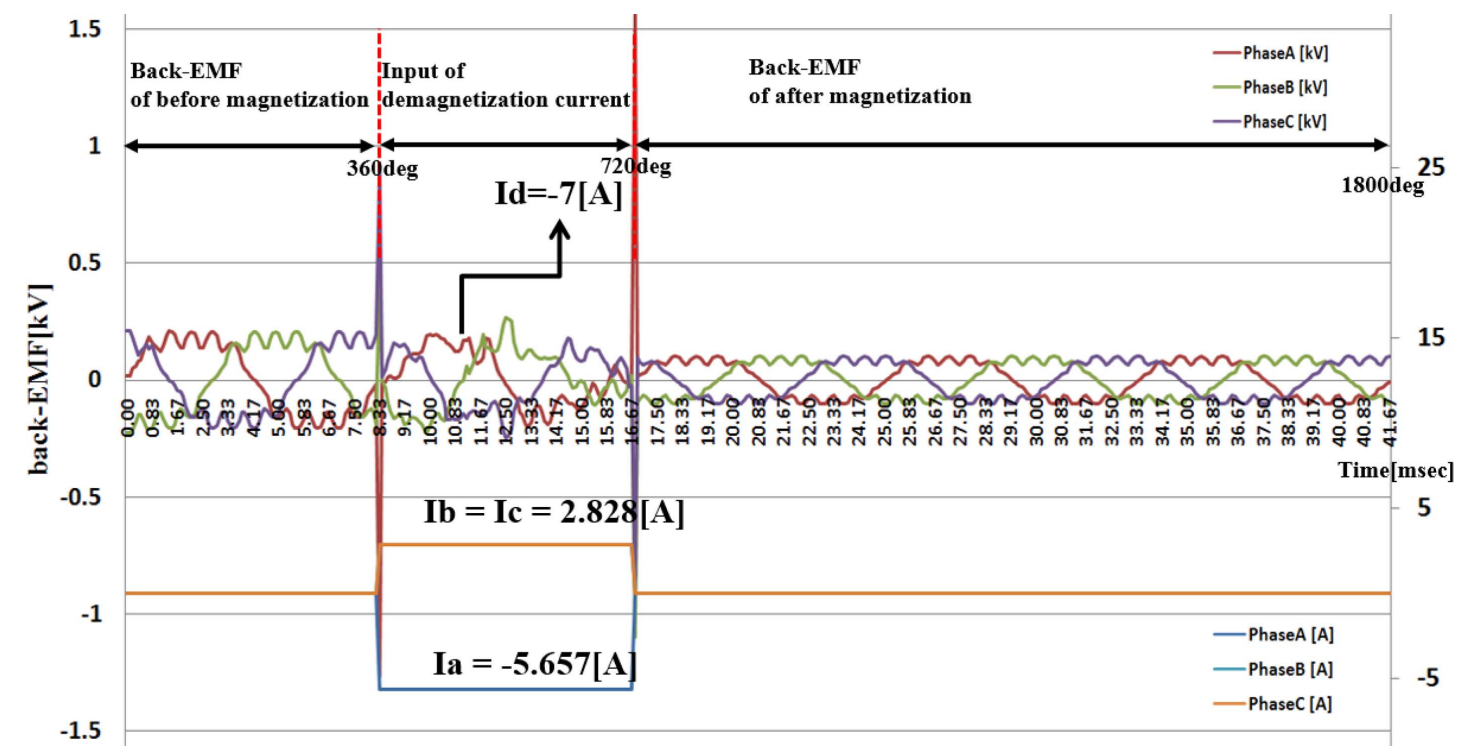

Fig. 7. (Color online) Back-EMF wave before and after the application of the d-axis current. 


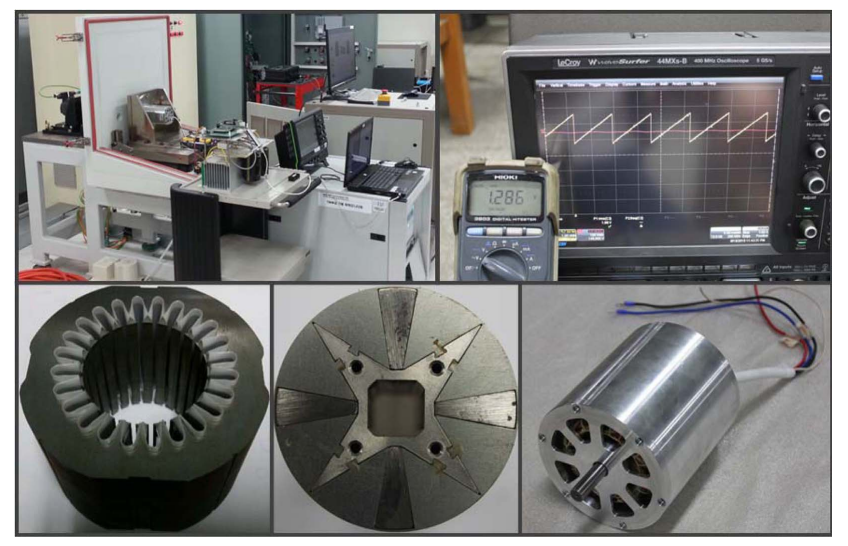

Fig. 8. (Color online) Photograph of VFMM and experiment equipment.

manent rotor, $I^{2} R$, in order to generate air-gap flux, whereas a VFMM can generate the flux almost without losses. This principle offers an attractive efficiency at high speed regions, because a field-weakening control and the d-axis currents are not needed.

In order to verify the above principle, a prototype of a VFMM was built. The motor and the installed TMS320F28335 DSP board inverter are shown in Fig. 8. The input current source used a PWM switching signal produced by an inverter. The generated PWM signal was applied to the motor via a vector control. The d-axis current, $i_{d}$, which flowed through the stator winding(s), were supplied from the same source as the stator current. The load current, $i_{q}$, did not have any effect on the magnetization of magnets.

\section{Comparison Results Between FEM and Experimentation}

Figure 9(a) and (b) show a comparison of the backEMF waves by the FEM analysis and the measured experimental results. Comparing the FEM results and the measured experimental results, the measured data is similar to the results of the FEM analysis. In this sense,

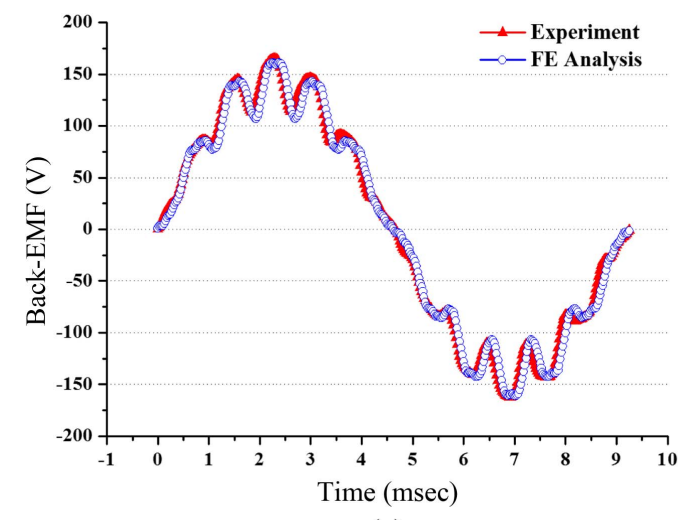

(a)

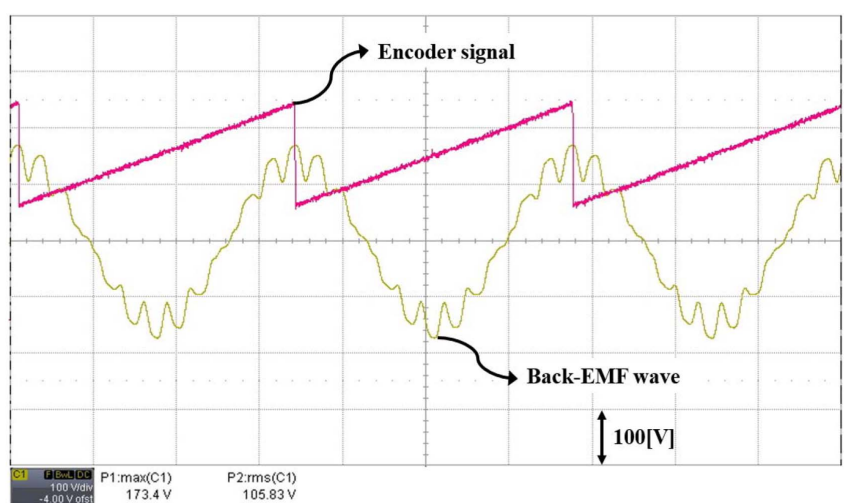

(b)

Fig. 9. (Color online) Back-EMF comparison with applied d-axis current (+40 A) between (a) FEM analysis result and (b) measured experiment result.

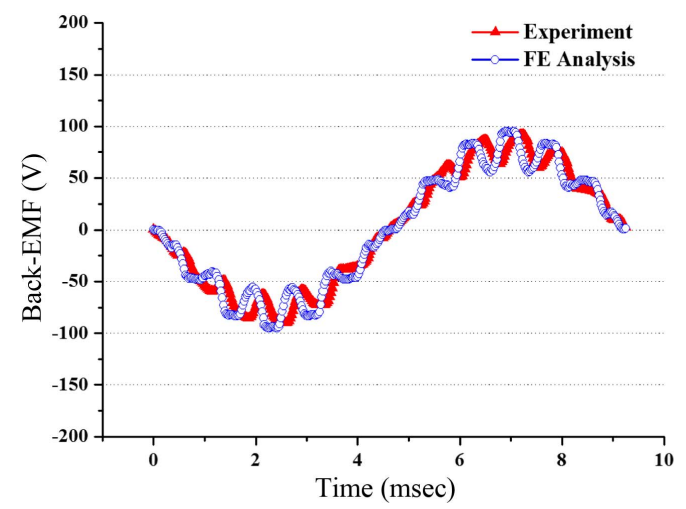

(a)

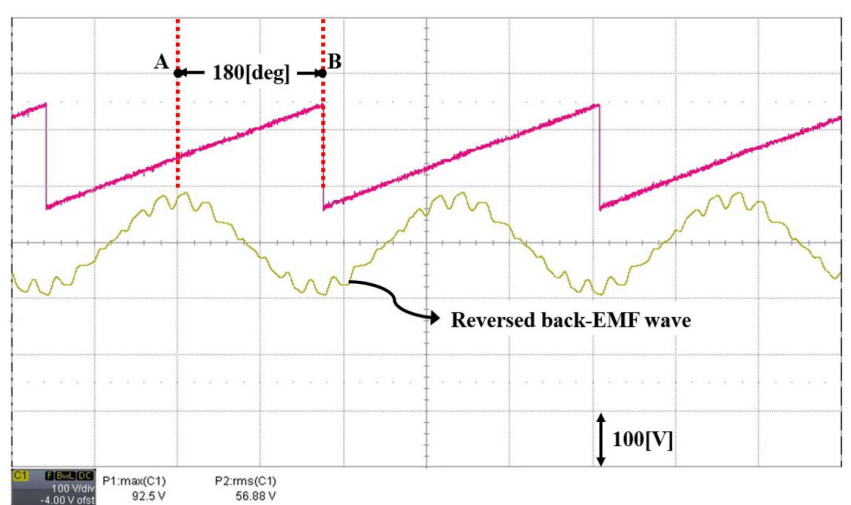

(b)

Fig. 10. (Color online) Measured back-EMF as applied d-axis current (-18 A). 
our FEM analysis is very reliable. In Fig. 9(b), encoder signals are used to determine the rotor position and can also be used to check variations of phase with respect to the intensity of the negative d-axis current.

As mentioned above, when a d-axis current of $-18 \mathrm{~A}$ is applied, the back-EMF wave would reverse, which is validated by the encoder signal being moved from the "A" point to the "B" point (in exactly $180^{\circ}$ ), as shown in Fig. 10.

\section{Conclusion}

The re- and de-magnetization characteristics of PMs and the back-EMF of a VFMM can be estimated by FEM, which is suitable for the evaluation of machines with magnetic non-linearity, hysteresis phenomena and magnetizations. When built with alnico magnets, a VFMM offers the ease of PM magnetization control. Thus, a VFMM combines the high efficiency of a wide-speed machine with the air-gap flux controllability of a wound rotor synchronous machine.

These results make the VFMM an optimal choice for many applications in industry, as they can be used as standard drives.

\section{Acknowledgment}

This research was supported by the research fund of Hanbat National University in 2012.

\section{References}

[1] S. Y. Jung, C. C. Mi, and K. H. Nam, IEEE Trans. Ind. Appl. 62, 3380 (2015).

[2] Jacob A. Krizan and Scott D. Sudhoff, IEEE Trans. Energy Conversion 28, 95 (2013).

[3] S. M. Sue and C. T. Pan, IEEE Trans. Ind. Appl. 55, 340 (2008).

[4] W. Hatsuse, Y. Notohara, K. Ohi, and K. Tobari, International Power Electronics Conference (2010) pp. 599-604.

[5] V. Ostovic, IEEE-IAS Annu. Meeting (2001) pp. 25772584.

[6] J. H. Lee and J. P. Hong, IEEE Trans. Magn. 44, 1550 (2008).

[7] Jacek. F. Gieras, CRC Press (2009) pp. 51-58. 